Horizontes. Revista de Investigación en Ciencias de la Educación

ISSN 2616-7964

octubre-diciembre, 2017

Volumen 1, Número 4

HORIZONTES

pp. 10-15

www.revistahorizontes.org

(c) (i) (2) (2)

\title{
La didáctica como tarea humanizadora de la praxis pedagógica en la Universidad Militar Bolivariana de Venezuela
}

\author{
Didactics as a humanizing task of pedagogical practice at the \\ Bolivarian Military University of Venezuela
}

\author{
Pedro Reinaldo Pérez Hurtado \\ reiper24@hotmail.com, ivanovishph@gmail.com \\ Universidad Bolivariana de Venezuela
}

Recibido: 09-04-2017 / Revisado: 13-04-2017 / Aceptado: 14-05-2017 / Publicado: 09-10-2017

\begin{abstract}
RESUMEN
La didáctica ha sido considerada, como una Ciencia teórico-normativa que guía de forma intencional el proceso optimizador de la enseñanza-aprendizaje, en un contexto determinado e interactivo, posibilitando la aprehensión de la cultura con el fin de conseguir el desarrollo integral del estudiante, cuya génesis data desde los griegos, pero en el siglo XVII, se hace evidente la necesidad de enunciar bases aplicables a la educación acordes al pensamiento filosófico de éste período y los grandes cambios políticos, religiosos, económicos, sociales y culturales. El Sistema Pedagógico Militar exige una praxis educativa que se ajuste a los procesos de cambio que se vienen dando en los ámbitos educativo, cultural y tecnológico a nivel nacional $e$ internacional. Por esta razón, es indispensable que las personas dedicadas a la docencia militar adquieran una sólida formación que dé respuesta a las nuevas demandas socio-educativas del estado en concordancia con los lineamientos educativos emanados por el Viceministerio de Educación para la Defensa y de los Componentes, tanto en los contextos científicos y culturales como en el plano didáctico, reflexionando sobre las modernas teorías y tendencias pedagógicas en el ámbito de su competencia.
\end{abstract}

Palabras clave: Didáctica, Sistema pedagógico militar, Educación, Praxis
ABSTRACT

The didactic has been considered, as a theoreticalnormative Science that intentionally guides the optimizing process of teaching-learning, in a determined and interactive context, making possible the apprehension of the culture in order to achieve the integral development of the student, whose genesis dates from the Greeks, but in the seventeenth century, it becomes clear the need to enunciate bases applicable to education according to the philosophical thought of this period and the major political, religious, economic, social and cultural changes. The Military Pedagogical System requires an educational praxis that adjusts to the processes of change that are taking place in the educational, cultural and technological fields at a national and international level. For this reason, it is essential that the people dedicated to military education acquire a solid formation that responds to the new socioeducational demands of the state in accordance with the educational guidelines emanating from the Vice Ministry of Education for Defense and Components, both in the scientific and cultural contexts and in the didactic level, reflecting on the modern theories and pedagogical tendencies in the field of their competence.

Key words: Teaching, Military educational system, Education, Praxis 


\section{INTRODUCCIÓN}

En el marco de reflexión sobre la Didáctica y fundamentalmente sobre la praxis de la misma en la Universidad Militar Bolivariana de Venezuela (UMBV), nos podemos plantear cuál ha sido el papel que ha jugado la didáctica y cuál es su importancia, en cuanto a la organización de políticas y recursos de apoyo efectivo a la docencia en el ámbito de la educación universitaria militar, cuáles han sido los obstáculos encontrados para el logro exitoso del proceso de enseñanza-aprendizaje- que a mi parecer existen una diversidad de concepciones sobre el significado de la misma y sus postulados teóricos-metodológicos en relación a la construcción y uso de técnicas didácticopedagógicas en los docentes de la UMBV, esto representa varias dificultades y retos, no sólo por tratarse de una reflexión pedagógica sobre la didáctica, sino también por algunas cuestiones inherentes a la relación misma que se quiere describir y analizar en este escrito.

En cuanto a lo primero, podría decirse que en la universidad militar justifica la incursión pedagógica en la medida en que toda didáctica subyace una concepción sobre la relación entre el docente-alumnos y contenidos, la cual se hace necesario explicitar lo mejor posible para hacer de las practica de enseñanza una serie de acciones lo más claramente planificada y consecuente, considerando que durante el proceso de enseñanza aprendizaje se encuentran obstáculos didácticos que pueden llevar al fracaso el proceso de aprendizaje.

Por ello la transformación del sistema educativo militar deberá establecerse con la conformación de una metodología participativa para reivindicar la enseñanza, ya que el problema didáctico se funda sobre el desconocimiento del comportamiento mental de los estudiantes en relación con el comportamiento del docente cuando elabora sus estrategias de enseñanzas- lo que denominamos - praxis dialógica- para la construcción colectiva del aprendizaje y el fortalecimiento de la profesionalidad de los docentes, y preguntarse sobre qué elementos permiten aplicar la didáctica en los ambientes de aprendizaje, bajo que criterios se describen los perfiles educacionales en el ámbito castrense. En consecuencia es fundamental establecer elementos didácticos que busquen enlazar el entorno del estudiante y la vida universitaria como un ámbito de conocimiento y comunicación que se ocupe del arte de enseñar, ya que evidentemente, quien no sabe bien, no puede enseñar bien, ya que un docente que no domina las estrategias didácticas, y deje de lado su importancia en el proceso de enseñanza-aprendizaje, no podría lograr en sus alumnos el desarrollo de competencias y habilidades para interpretar la realidad, o analizar los fenómenos socioculturales de su entorno. De tal modo que la formación didáctica no puede ser superflua. Ella debería enfatizar sobre el siguiente trípode: lo afectivo-cognitivo-praxiológico, de manera que cada ser se prepare, a la vez, para saber, hacer y convivir, condiciones que se demuestran en la praxis del desempeño pedagógico y en el debido uso de las estrategias didácticas de los profesores.

En consecuencia, educar no es sólo enseñar a aprender, no es sólo instruir, no es sólo enseñar a conocer; no es ni siquiera "hacer que los estudiantes sean más buenos", más originales y creativos, más sensibles a los otros o mejores ciudadanos. Todo ello, sin duda, es un elemento clave para la formación del hombre nuevo, que quiere formar la universidad militar, quien tiene como fundamento educativo el desarrollo de las capacidades físicas y psíquicas de los integrantes de la fuerza armada nacional bolivariana, a través de procesos educativos sustentados en valores, garantes de la praxis del nuevo profesional militar. De tal manera como señala Herrán (2010). Si se parte de que enseñar bien es lo que se hace habitualmente, no podemos más que señalar que el ser humano tiene una enorme capacidad para valorar positivamente lo malo o lo mejorable, 
con tal de que dure el tiempo necesario. Ya que según él los medios de comunicación manejan la didáctica muy bien al igual que los programadores de TV y las casas de editoras de discos. Sabemos que esto es algo constatable en la cotidianidad de nuestra sociedad, y, desde luego, desde la de tantos estudiantes universitarios. Porque, ¿cuántas veces se ha dado el caso de excelentes profesores, de los que se ha reconocido, además de su conocimiento, su incompetencia didáctica? Que a mi parecer esto podría cambiarse si la universidad militar pasara por un proceso de Introducción Didáctica, para transformar a los alumnos -partiendo que la didáctica es propia del docente- en agentes críticos inductores de cambio, directamente en ellos, es decir los estudiantes, e indirectamente en sus docentes. De hecho, no debería ser no pertinente reflexionar sobre aquello que, desde el punto de vista de la comunicación, viven día a día, y de lo que podría depender un mejor proceso de enseñanza-aprendizaje.

El debate sobre la didáctica universitaria, especialmente en la UMBV debe contemplar la práctica pedagógica como un espacio del quehacer comunitario; donde el docente debe estar comprometido con el nuevo pensamiento militar; la asunción del Estado Docente como doctrina en el marco de la concepción del Estado Bolivariano para garantizar la educación como un derecho humano fundamental, basado en el concepto de corresponsabilidad en el ámbito de la defensa nacional. El proceso de modernización del currículo de la Universidad Militar Bolivariana de Venezuela (UMBV) se desarrolla a partir de propuestas claves que aseguran su adecuada prosecución y el desarrollo de la personalidad de los alumnos y las alumnas en igualdad y sin exclusiones de ningún tipo y la formación en y para la convivencia democrática.

\section{METODOLOGÍA}

De allí que se puede proponer una didáctica militar cuya episteme este enmarcado en el desarrollo del conocimiento científico y humanístico aplicado a las Ciencias y Artes Militares. Esta metodología debe ser producto de una amplia revisión los conceptos pedagógicos y de los elementos didácticos conceptualizados en los diversos documentos que describen el modelo de la nueva doctrina militar, que según Aguana (2014) garantiza la formación de un nuevo ser social militar, capaz de incidir, crear y transformar su contexto-sociocultural, consolidar el pensamiento militar venezolano y la unión cívico-militar.

\section{DISCUSIÓN}

De acuerdo a lo planteado la universidad militar debe hacer una autorreflexión desde la praxis pedagógica y la didáctica como un reto que permita ver de dónde venimos y hacia dónde va el desarrollo de las competencias explicitas en el currículo y la pertinencia de las estrategias de aprendizaje que se bien aplicando en la misma, tal como lo plantea Aguana (2012) se precisa asumir concepciones y modelos formativos en lo cívico-militar en correspondencia con las características que expresan el nuevo Estado, a partir de un reconocimiento del valor del contexto político-social desde su diversidad dinámica y cambiante, que propicie un desempeño flexible, comprometido, competente $\mathrm{y}$ trascendente de quienes defienden la soberanía nacional y la identidad del pueblo de tal modo que se constituye en una dialéctica que abarcara lo comunicativo en todos los ámbitos de la sociedad.

Por ello la reflexión de Aguana (2012) respecto al modelo educativo militar hace énfasis en que éste debe estar orientado a la obtención de conocimientos, habilidades, actitudes, valores éticos-morales, entre otros, a partir de contenidos referidos al perfil militar profesional, lo que constituye el proceso de transferencia de conocimientos, habilidades, valores los cuales se convierten necesariamente en la directriz esencial a lo largo de su carrera militar, fundamentado en estrategias didácticas propias de una 
educación emancipadora para la Fuerza Armada Nacional bolivariana, la cual tiene que ser inédita y original.

Por lo tanto, tal como lo señala Aguana (2012) habrá de considerarse el proceso formativo militar como el eslabón donde se adquieren los contenidos culturales militares indispensables para ejercer la profesión, sin embargo el carácter formativo militar requiere de profundos análisis para desentrañar relaciones importantes que permitan considerar a la formación militar como verdadero proceso humano, a partir de la concretización de los perfiles de formación de los futuros profesionales militares y sea desde allí vincularte con su ser profesional por ello es urgente acordar una propuesta de enseñanza que incite a los estudiantes a cuestionar y desafiar las creencias y prácticas que se les imparten con el fin de promover una conciencia crítica a la luz de una didáctica universitaria acorde al ámbito castrense.

La didáctica universitaria como praxis humanizadora del conocimiento en la Universidad Militar Bolivariana.

A quince años delas reformas de educación universitaria, la Universidad Militar Bolivariana de Venezuela se enfrenta a grandes desafíos y dificultades, relacionados con la praxis didáctica y la formación de sus profesionales, la misma debe estar basada en competencias, y pertinencia con los planes de estudios, orientadas al desempeño laboral de los egresados y egresadas de los centros educativos adscritos a la UMBV, que de igual manera, debe hacer frente a los retos que suponen las nuevas oportunidades de los avances tecnológicos que de alguna amanera mejoran las formas de producir, organizar, difundir y controlar el saber y de acceso al mismo.

En tal sentido, se puede afirmar que la didáctica desarrollada en el Sistema Pedagógico Militar, está dada por un horizonte de formación que busca persuadir mediante la retórica- en sus nuevas modalidades- a los sujetos de un conjunto de valores. Este proyecto que se lleva a cabo intersubjetivamente en la UMBV, busca entre objetivos, la construcción de una identidad propia en la práctica pedagógica en el sistema universitario militar.

En este orden de ideas y en aras de encontrar soluciones para estos desafíos y de poner en marcha un proceso de profunda reforma de la educación militar debemos comenzar por conceptualizar la práctica didáctica, definición de los perfiles de egreso en sus diseños curriculares, los programas y planes de estudios que como institución de educación universitaria debe asegurarse que los proyectos y programas de formación de sus oficiales, respondan al desarrollo científico, tecnológico y humanístico, es decir, a los elementos onto-epistemológicos que sustentan el sistema educativo universitario y en especial al militar para lo cual se requiere evaluar la oferta académica y coordinar los procesos de transformaciones curriculares y formular las instrucciones de renovación técnica de acuerdo con los objetivos del Reglamento Educativo Militar (2010),donde se describen en que consiste la planificación curricular de cada centro de educación militar, y qué estrategia didácticas definen los medios e instrumentos necesarios para el logro de los fines institucionales. Respondiendo a las exigencias y demandas de la sociedad para redefinir sus fines al servicio de la sociedad.

\section{La didáctica como eje de transversalidad en el Currículo de la Universidad Militar}

Para revisar qué modelo didáctico y la concepción educativa que sustentan el diseño del currículo de la Universidad Militar Bolivariana de Venezuela (UMBV), es necesario involucrarse y comprometerse con el Modelo y la filosofía educativa de la Universidad Militar y con los procesos de cambios que vive el país. Con ello se quiere resaltar el espacio que debe propiciar la reflexión e integración al diseño curricular los ejes transversales, "los mismos constituyen 
los fundamentos para la práctica pedagógica al integrar los campos del ser, el saber, el hacer y convivir a través de los conceptos, procesos, valores y actitudes que orientan la enseñanza y el aprendizaje, es importante destacar que los ejes transversales no son contenidos paralelos a las áreas sino medios que conducen a un aprendizaje que propicia la formación científico-humanística y éticomoral de un ser humano, cuyo logros y alcances se logran con una práctica didáctica eficiente y cónsona con los cambios sociales que se suscitan en cada periodo histórico en el cual surge la universidad. Por ello es necesario "volver a pensar" y "volver a crear" la Educación Universitaria Militar, para garantizar que la misma responda a las necesidades de la sociedad del siglo XXI, al momento histórico que vive la nación, a las exigencias de la sociedad y del conocimiento, así como a la existencia de una demanda real de la participación de la educación en los planes de desarrollos orientados a la excelencia y consolidación de una democracia participativa, solidaria y protagónica.

Se plantea un esquema curricular, donde la práctica didáctica este basado en el desarrollo de competencias, con énfasis en praxis de los valores, de tal manera que su objetivo sea destacar la magnitud e importancia en el desarrollo de los perfiles profesionales, y constituya la representación de las exigencias de carácter profesionalizante que se demandan en el mercado laboral $u$ ocupacional a los egresados de las instituciones de educación universitaria. Este debe hacerse evidente en los planes de estudio mediante la formulación categorizada de rasgos, conocimientos, habilidades, destrezas que deberán adquirirse y desarrollarse durante su formación.

Sin embargo, la universidad podría constituirse en el vínculo válido sobre todo para que sus estudiantes adquieran una visión más amplia de lo que ocurre y de lo que tenemos entre manos, radicada en el humanismo necesario. Si la universidad es un sistema organizado de profesionales, básicamente, estudiantes y docentes, sus posibilidades de cambio radican, primero, en la mentalidad de sus agentes personales, y después como consecuencia lógica de ello, en la organización de su oferta formativa y en su didáctica universitaria. De ello deducimos que la mentalidad siempre tiende a ir por delante, y el apego a la organización tradicional puede ser un factor de obstáculo de la renovación más crítica de la práctica didáctica.

\section{CONCLUSIÓN}

La universidad, o es o no se diferenciará formalmente de otra clase de instituciones productivas. A partir de su organización, no es extraño que la formación que promueve quede condicionada por ella, a pesar de que el nuevo paradigma constructivista se enfrente al paradigma conductista, por ello es urgente que se implemente un programa de Formación Didáctica Universitaria: que favorezca todo lo anterior, centrando la enseñanza en la reflexión, en el desarrollo de competencias y la producción creativa, como una parte esencial de la profesionalidad del docente universitario. Pero para acceder a esos cambios, la universidad militar tiene ir más allá de una formación didáctica universitaria, basada en la ignorancia; romper con los modelos de proyectos didácticos individualistas. Debe ir más allá del encastillamiento de las áreas del conocimiento, que cada vez menos soportan la realidad que las desborda; el espíritu verdaderamente cooperativo y el trabajo en equipo, más allá de los egocentrismos comunitarios y sus micro intereses, a veces microcéfalos, porque olvidan o relegan al estudiante; la investigación en didáctica, deberá ir más allá del abandono metodológico; deberá promover la originalidad, la orientación y tutoría universitaria, debe estar por encima de la sola-instrucción; la autocrítica personal e institucional, más allá de las vigas de ojos ajenos; las alturas personales y humanas, más allá de la técnica; y quizá por último, el deseo 
de madurez profesional más allá de ser un buen docente.

\section{REFERENCIAS}

Aguana, Rafael José (2012). La militaridad en el Estado democrático y social de derecho y de justicia. 2da edición. Caracas, Venezuela: Universidad Militar Bolivariana de Venezuela.

Constitución de la República Bolivariana de Venezuela (1999): Gaceta Oficial № 36.860. Imprenta Nacional. Caracas, Venezuela

González L., Fernando (2001). La educación como tarea humanizadora. Madrid, España: Grupo Anaya,

Herrán, A. (2010). Disparates pedagógicos o retos de la enseñanza universitaria. En J. Paredes y A. de la Herrán (Coords.), Cómo enseñar en el aula universitaria. Madrid, España: Pirámide

Malpica B, Federico (2012): Referentes para la transformación de la práctica en la enseñanza y aprendizaje por competencias. Alianza Educativa. México.
Miller, (2000) El pensamiento complejo y la pedagogía. Bases para una teoría holística de la educación. Citado en Estudios Pedagógicos, № 26, pp. 133-148

Monereo, C. y Pozo, J.I. (2003) (edit.). La Universidad ante la nueva cultura educativa. Enseñar y aprender para la autonomía. Madrid, España: Síntesis.

Páez, C. (2006). Los modelos didácticos. Disponible en http://carmenps2.wordpress.com/2006/ 12/11/modelos-didacticos (Consultado el 3 de Noviembre de 2009).

Rodríguez, A., Caurcel, M.J. y Ramos, A.M. (2008). Didáctica en el espacio europeo de Educación Superior. Guía de trabajo autónomo. Madrid, España

Universidad Militar Bolivariana de Venezuela (2011). Documento Rector. 